\title{
Dissolution Highlights from the 2015 AAPS Annual Meeting in Orlando
}

\author{
Nikoletta Fotaki ${ }^{1,}{ }^{*}$, Vivian Gray ${ }^{2}$, Johannes Krämer ${ }^{3}$, Dorys Diaz ${ }^{4}$, \\ Talia Flanagan ${ }^{5}$, and Geoffrey Grove ${ }^{6}$ \\ ${ }^{1}$ Department of Pharmacy and Pharmacology, University of Bath, Claverton Down, Bath, BA2 7AY, UK \\ ${ }^{2}$ Dissolution Technologies, Hockessin, DE, USA \\ ${ }^{3}$ Phast, Homburg, Germany \\ ${ }^{4}$ Worldwide Research and Development, Global Chemistry and Manufacturing Controls, Pfizer Inc, Eastern Point Road, Groton, CT, USA \\ ${ }^{5}$ Astra Zeneca, Macclesfield, UK \\ ${ }^{6}$ Sotax, Westborough, MA, USA
}

A APS held its Annual Meeting and Exposition at the Convention Center in Orlando, Florida, during the week of October 25-29, 2015. The meeting is a premier gathering of pharmaceutical scientists from around the world and works to address the needs of the attendees, including members of over 40 focus groups in nine sections, or scientific disciplines, including two focused on dissolution testing: the In Vitro Release and Dissolution Testing and QbD and Product Performance groups.

For those with an interest in dissolution testing, there was a preconference short course on "Global Criteria for Biowaiver of Various Dosage Strengths of a Product" and during the conference, two sunrise sessions on "Dissolution Similarity Requirements: How Similar or Dissimilar Are the Global Regulatory Expectations?" and "Systematic Design of In Vitro Testing Methods for Drug Product Development" and two roundtable sessions on "Regulatory Mismatch Between the USP and JP in the Dissolution Testing of Capsule Products" and "Clinically Relevant Specifications: Where Are We Now?" There were face-to-face meetings of the two focus groups mentioned and the Dissolution Discussion Group.

\section{SHORT COURSE: GLOBAL CRITERIA FOR BIOWAIVER OF VARIOUS DOSAGE STRENGTHS OF A PRODUCT}

This one-day preconference short course was organized and moderated by Barbara Davit (Merck, USA) and Yu Chung Tsang (Apotex, USA). It started with a presentation from April Braddy (FDA, USA) titled "U. S. Requirements for Bioequivalence Studies and Biowaivers Criteria for Different Strengths of an IR or MR Product Containing Single or Multiple Active Ingredients." A brief history of the benefits of generic products in the United States was given. She outlined the present U.S. regulations and guidelines for the generic industry, including the pertinent parts of the Code of Federal Regulations (CFR), 21 CFR
320.24 (b), and the Guidance "Bioequivalent Studies with Pharmacokinetics Endpoints for Drug Submitted Under an ANDA." Dr. Braddy described the bioequivalence (BE) study designs for moieties, in particular, enantiomers and racemates. The BE criteria for biowaivers for additional strengths for IR products were discussed. She emphasized that the formulations have to be proportionally similar across all strengths and cautioned for fixed-dose combinations. The non-applicability to narrow therapeutic index drugs and those drugs that are absorbed in the oral cavity was noted. Reference was made to the revised draft guidance "Waiver of In Vitro Bioavailability and Bioequivalence Studies for ImmediateRelease Solid Oral Dosage Forms Based on the Biopharmaceutics Classification System" and the new guidance "Dissolution Testing and Specifications Criteria for Immediate-Release Solid Oral Dosage Forms Containing Biopharmaceutics Classification System Class 1 and 3 Drugs." Modified-release (MR) products criterion for additional strengths and the additional in vitro studies needed for detection of dose dumping were described. Special topics of complex mixtures and endogenous compounds were explored. She informed the participants on the collaboration efforts of the International Generic Drug Regulators Programme (GDRP) and a review article titled, "International Guidelines for Bioequivalence of Systemically Available Orally Administered Generic Drug Products: A Survey of Similarities and Differences." The second presentation on "Canadian Requirements for Bioequivalence Studies and Biowaiver Criteria for Different Strengths of an IR or MR Product Containing Single of Multiple Active Ingredients" was given by Paul Wielowieyski (Health Canada, Canada). The BE guidance document used in Canada is "Conduct and Analysis of Comparative Bioavailability Studies and Comparative Bioavailability Standards: Formulations Used for Systemic Effects." The modifications to the BE requirement that deal with critical-dose, early onset, and highly variable

${ }^{*}$ Corresponding author. 
drug products and the pharmacokinetic considerations of testing different product strengths in vivo were discussed. The in vitro approaches to demonstrate equivalence are addressed in "Bioequivalence of Proportional Formulations-Solid Oral Dosage Forms" and "Biopharmaceutics Classification System Based Biowaiver." He talked about the biowaiver conditions for proportional formulations, the limits for excipient classes, bracketing and comparative dissolution testing conditions. Dr. Wielowieyski discussed proportional formulations as applied to MR products and fixed-dose combinations and continued the presentation with eligibility characteristics for BCS-based biowaivers. He concluded his talk with the steps toward global harmonization, which included topics of BE standards for highly variable drugs, fed-state studies instead of fastedstate studies, and waivers for drug products containing both Class 1 and 3 drug substances. Ivana Taševská (State Institute for Drug Control) talked about the "EU Requirements for Bioequivalence Studies and Biowaiver Criteria for Different Strengths of an IR or MR Product Containing Single of Multiple Active Ingredients." She reviewed the EU guidances connected to $\mathrm{BE}$ and biowaivers and went through several questions and answers on biowaiver topics. The first topic was related to the rules applied to $f_{2}$ calculations, and caution against using the Mahalanobis distance if $f_{2}$ criteria are not met was emphasized. The use of a model-independent method is most suitable for profile comparison, and the use of a combined approach for reporting $f_{2}$ along with $A U C$ or dissolution efficiency $(D E)$ values as quality indicators was recommended. Bioequivalence of gastroresistant preparations was discussed, and the recommendation of using tight sampling intervals even if more than $85 \%$ is dissolved before 15 min was emphasized. She also advised reading the $Q$ and $A$ sections of these guidances for some critical information. She concluded her presentation with the statement that the influence of excipients on product performance is not negligible. The fourth presentation on "Formulation-Related Factors that Can Affect Biowaivers for Different Strengths of a Product-Challenges on Modified-Release Formulations and Combination Products" was delivered by Shrinivas Murti (Merck, USA). He started his talk by reviewing the biowaiver fundamentals for multiple-strength products. He discussed proportionality similarity with fixed-dose combinations (FDC), drawing on the FDA BE guidance and the EMA view, with an emphasis on bilayer tablets. Afterward, he talked about strategic considerations for FDC including APIs with unique properties, formulation interactions, potentially more restrictive biowaivers, and lack of predictive preclinical in vitro and in vivo models.
He continued with the issues for FDC surrounding the number of strengths developed and layered tablets. The topic of MR formulations was explored. The considerations for MR are driven by clinical and technology issues, restrictive biowaivers, and the strong regulatory preference for establishing a Level A IVIVC. Case studies on bridging strategies for FDC were presented. He concluded with a discussion of previous FDC strategies that are no longer globally viable. Nikoletta Fotaki (University of Bath, UK) gave a presentation titled "Analytical and Dissolution Requirements in Assuring Bioequivalence of Different Strengths." She highlighted the importance of in vitro dissolution testing as a guiding technology during drug product development. Differences in the dosage forms performance from step to step are indicated by discriminative dissolution methods. The use of dissolution methodology for quality control (QC) testing requires discriminative power in an ideal case proven by in vitro-in vivo correlation (IVIVC). This link between in vitro and in vivo performance of dosage forms is pivotal for claiming a biowaiver based on in vitro data. She cited the recently issued European guideline for modified-release dosage forms (EMA/CHMP/ QWP/428693/2013), which requires establishing a link from in vitro release to pharmacokinetic parameters. She described steps beyond the selection of the apparatus for dissolution media including the $\mathrm{pH}$ range and the addition of surfactants by referring to the updated USP General Chapter <1092> for the proper concentration of surfactants. Afterward, she expanded her European perspective to immediate-release (IR) dosage forms. The solubility and permeability of the drug substance are the major criteria for the categorization according to the Biopharmaceutics Classification System (BCS) as a tool to link the in vitro to the in vivo performance. As she exemplified for BCS Class 1 and 3, the prerequisites for claiming a biowaiver for different dosage strengths include the sameness of the manufacturing process, a proportional composition, less than $5 \%$ of drug substance referred to the drug product, and similar dissolution profiles. Similarity of in vitro dissolution is given if not less than $85 \%$ of label claim is dissolved in 15 min for test and reference formulations. In other cases, the $f_{2}$ should be applied provided that $85 \%$ of the drug substance is dissolved in 30 min. Nagesh Bandi (Pfizer, USA) gave the last presentation of the short course on "Challenges in Developing Global Formulations for Submissions to Various Jurisdictions-Needs for Harmonization." Besides the attractive markets governed by the internationally harmonized quality guidance (International Conference on Harmonization of Technical Requirements for Registration of Pharmaceuticals for Human Use), other 
parts of the world evolve to target markets for the globally acting pharmaceutical companies. He gave an overview on the biowaiver requirements for countries such as South Africa, Thailand, Turkey, Korea, Australia, Mexico, and India. He put emphasis on the BA/BE (bioavailability/ bioequivalence) criteria. In general, for solid oral dosage forms the proof of $\mathrm{BE}$ is required for the highest dosage strength. A waiver for a lower strength can be obtained in the case of linear pharmacokinetics, proportional composition, and similar in vitro dissolution behavior. The dissolution profiles used for a similarity check should be obtained from the classical USP basket (50-100 rpm) or paddle (50-75 rpm) apparatus. Media should be aqueous buffer solutions adjusted to $\mathrm{pH}$ values between 1.2 and 7.5. In Japan, the use of dissolution data is limited by levels of compositional differences within a row of various dosage strengths. The requirements are common for IR and MR dosage forms. To prove dissolution similarity, Japanese authorities require the same total dose tested for both of the formulations (e.g., two 50-mg tablets dissolved under the same conditions as one 100-mg tablet per vessel). The amount of dissolution testing under the default method or extended to various other dissolution conditions depends on the level of change. Interestingly, some countries have additional requirements for in vitro dissolution testing (e.g., in Turkey, the description of in vitro dissolution kinetics requires the use of at least six data points for calculation of $f_{2}$ ). An overview of how $f_{2}$ is applied was presented (Table 1).

Table 1. Rules for $f_{2}$ in Different Countries

\begin{tabular}{|l|l|}
\hline Country & Coefficient of Variation Criteria \\
\hline $\begin{array}{l}\text { United States, } \\
\text { Canada, South Africa, } \\
\text { Brazil }\end{array}$ & $\begin{array}{l}\text { The percent coefficient of variation at the } \\
\text { earlier time points should not be more than } \\
20 \%, \text { and at other time points not be more than } \\
10 \% .\end{array}$ \\
& $\begin{array}{l}\text { For Brazil, the first 40\% related time points are } \\
\text { considered "earlier time points." }\end{array}$ \\
\hline $\begin{array}{l}\text { EU, Australia, Russia, } \\
\text { China, Mexico, } \\
\text { Turkey }\end{array}$ & $\begin{array}{l}\text { The percent coefficient of variation at the first } \\
\text { time point should not be more than } 20 \%, \text { and } \\
\text { at other time points not be more than 10\%. }\end{array}$ \\
\hline Korea & $\begin{array}{l}\text { The percent coefficient of variation should not } \\
\text { be more than 15\% at all time points. }\end{array}$ \\
\hline Thailand & $\begin{array}{l}\text { The percent coefficient of variation should not } \\
\text { be more than 10\% from second-to-last time } \\
\text { points. }\end{array}$ \\
\hline
\end{tabular}

The short course was concluded by a panel discussion, where it was mentioned that the European Pharmacopoeia (Ph. Eur.) will have monographs on formulations similar to those in the USP. Barbara Davit led the discussion and Dissolution

Technologies MAY 2016 contributed her documented experience in the field of in vivo performance testing (1).

\section{SUNRISE SESSION: DISSOLUTION SIMILARITY REQUIREMENTS: HOW SIMILAR OR DISSIMILAR ARE THE GLOBAL REGULATORY EXPECTATIONS?}

This sunrise session was organized by Dorys Argelia Diaz (Pfizer, USA) and was designed to stimulate discussion on the regulatory requirements to meet countryspecific regulatory expectations for dissolution profile similarity assessment, present industry perspectives on challenges and opportunities, and advance the discussion on the importance of harmonization of dissolution requirements. Global regulatory requirements for dissolution profile similarity divergence are on the rise. The rising challenge of global suitability of studies using $f_{2}$ to compare dissolution profiles was discussed as well as country-specific bioequivalence guidelines.

Vivian Gray (V.A.GrayConsulting, USA) gavea presentation titled "In Vitro Equivalence-Is Harmonization Possible?" that focused on the necessity to harmonize dissolution guidances to enable timely access to medicines. She discussed the globally divergent regulatory requirements for bioequivalence, multipurpose dissolution guidances, and the complexity of global dissolution filing strategies, along with the impact on research, innovation, and access to medicines (2). She provided business case examples along with a powerful analysis of the multipurpose applicability of dissolution guidance explaining how complexity increases when referral to various guidance documents (e.g., method development, post-approval changes, biowaiver for additional strengths) is needed. She concluded by highlighting how the similarity $f_{2}$ statistical comparison is accepted globally and how subtle differences in country-to-country criteria and a proliferation of new countries with similarity requirement guidances are observed.

Ganapathy Mohan (Merck, USA) started his presentation with the drivers for developing a dissolution method including regulatory requirements, QC test, surrogate for in vivo release testing, and support of clinically relevant specifications. He discussed in detail specification setting as well as the current struggle associated with accelerating timelines and incomplete understanding of the manufacturing processes and dissolution methods. He also highlighted the risk of not meeting $f_{2}$ for postapproval changes and the significant dissolution-related queries received from health authorities, especially FDA and EMA. He shared a summary of dissolution 
queries related to specification setting $Q$, dissolution discriminating capability, the use of enzymes, surfactant selection and justification, rotation speed, $f_{2}$ calculation, and reporting format. He concluded by emphasizing the importance of clinically relevant specifications and encouraged a dialog toward harmonization of dissolution requirements. During the Question and Answer session, Dorys Diaz summarized the efforts that have been initiated to address the technical and regulatory gaps identified by the speakers. She also reported on the other steps initiated toward raising awareness of dissolution guidance harmonization efforts including publications, posters, and the AAPS sunrise session. For further action, Dorys pointed to the identification of other dissolution requirements that need to be harmonized, the potential regulatory challenges that should be considered, and the initiation of collaboration between industry and academia.

\section{SUNRISE SESSION: SYSTEMATIC DESIGN OF IN VITRO TESTING METHODS FOR DRUG PRODUCT DEVELOPMENT}

This sunrise session was organized and moderated by Alger Salt (GSK, USA) and Nikoletta Fotaki (University of Bath, UK). Systematic processes and strategies for in vitro test methods and appropriate specifications to assess quality and to predict in vivo performance of oral immediate-, modified-, and extended-release products are needed for efficient progression of assets through drug product development. Different dissolution test methods are needed to meet different objectives such as detecting changes in product performance due to changes in manufacturing conditions, predicting in vivo performance via biopredictive methods with clinically relevant specifications, and detecting changes in product performance caused by exposure to humidity, heat, and light throughout a stability program. This session was focused on strategies for developing methods and specifications to meet the different purposes stated above. The session started with a presentation from Sandra Suarez (FDA, USA) on "Strategies for Developing Dissolution Test Methods Fit for Purpose-QbD Case Studies." She described the purpose of Quality by Design in the pharmaceutical industry and the role of dissolution testing in QbD. Afterward she presented the FDA review experience on the strategies for developing dissolution methods under $\mathrm{QbD}$ containing regulatory applications. Two case studies were presented. The first case study illustrated the advantages of integrating biopharmaceutics tools in the early phase of drug development (QbD-based). In the second case study, the use of in silico modeling and simulation as an aid in developing a dissolution method fitted for purpose was described. She emphasized that the dissolution method discriminating ability and biopredictive power should be confirmed and discussed with the agency before making a decision on its use in the design space. The discriminatory ability of the dissolution method should be determined by the dissolution acceptance criteria and the time point apart from the method selected. The lack of data linking the critical attributes, dissolution and bioavailability, and bioequivalence in vivo may lead to design spaces that are not acceptable. She pointed out that in silico PBPK models are a promising tool in the development of dissolution methods fitted for purpose and in the determination of the critical attributes. She concluded her presentation by noting that the FDA encourages the development of biopredictive dissolution methods via the construction of IVIVR and IVIVC. The second presentation in this session on "A Strategy for Dissolution Method Development: A Risk-Based QbD Approach" was delivered by Danna Mattocks (Tergus Pharma, USA). She explored the reasons why a dissolution method development strategy is needed. Dissolution expectations of external regulators and internal customers are evolving. Afterward she described the current state of dissolution in terms of its use as a QC batch release test and the trend over the last 15 years for the use of biorelevant dissolution methods. The unsuitability of traditional dissolution methods for complex products (i.e., supersaturated systems, complex modified-release mechanisms) was highlighted. The future state of dissolution was described, and she pointed out that the key to success is the selection of a dissolution method that discriminates for the correct critical quality parameters. The risk of inadequately understanding the product and the benefits of a QbD approach for the dissolution method were described. An ideal dissolution method development strategy was presented as part of a wider biopharmaceutics approach including in vivo, in vitro, and in silico components. The key strategy principles are (1) a cross-functional collaboration to identify product critical quality attributes, (2) a tailored use of biorelevant dissolution techniques for formulation development, product understanding, identification of critical quality attributes and clinical continuity, (3) a final QC method to be based on biorelevant methods, and (4) a final QC method to discriminate for relevant critical quality attributes. Suggestions on maximizing the power of biorelevant methods were given. Advantages of dissolution profile modeling were described. She concluded her presentation by highlighting that discriminating and clinically relevant methods will enable regulatory approval and reduce the failures of bioavailability and bioequivalence studies.

Dissolution 
ROUNDTABLE: REGULATORY MISMATCH

BETWEEN THE USP AND JP IN THE DISSOLUTION TESTING OF CAPSULE PRODUCTS

$A$ roundtable on the regulatory mismatch between the USP and JP regarding dissolution testing of capsule products was organized by Geoffrey Grove (Sotax, USA). The focus of this session was on the use of enzymes in capsule testing. Currently, the USP allows for the use of enzymes, while the JP does not. The two presenters for this session were Margaret Marques (USP, USA), who represented the USP point of view, and Raymond Skwierczynski (Takeda Pharmaceuticals International, USA) representing the JP point of view. Dr. Marques's talk, titled "Recent USP Stimuli Article and Revision to $<711>$ Expanding the Use of Enzymes for Dissolution Testing of Capsules with Cross-Linking," focused on the recent revisions to Chapter $\langle 711\rangle$, which expand the use of enzymes for capsule testing by adding two new enzymes, papain and bromelain, to cover the $\mathrm{pH}$ range between 4.0 and 6.8. Her talk included both a technical review of the reasoning behind the choice to expand the use of enzymes, as well as an encouragement to all who attended to participate in the USP review process by reading and commenting on stimuli articles published in USP Pharmaceutical Forum. It was made clear that the current USP position is that the use of enzymes for capsule testing assumes that the test is being performed as a manufacturing QC test only and that no relationship to in vivo results should be inferred. Dr. Skwierczynski's talk, titled "Scientific Basis the JP Position that Enzymes Should Not Be Used in Capsule Testing," covered a review of biostudy results showing physiological differences due to capsule cross-linking in certain patient populations. The data he reviewed showed that for populations with lower stomach acid levels, a reduction in the $C_{\max }$ was observed. His talk also included estimates of the level of hypochlorhydria in several global populations with greater than $20 \%$ in a number of countries. During the session, it was also mentioned that although no data are available for polypharmacy and geriatric patients, it stands to reason, based on the results of the current biostudy, that these populations may also be susceptible to the same drug performance issues. This raises a final question: should the FDA prohibit the use of enzymes in capsule dissolution testing, much like the recent FDA guidance for BCS Class 1 and 3 drug testing, when the "time to maximum plasma concentration is critical to the intended use"?
ROUNDTABLE: CLINICALLY RELEVANT SPECIFICATIONS: WHERE ARE WE NOW?

A roundtable titled "Clinically Relevant Specifications: Where Are We Now?" was organized and moderated by Talia Flanagan (Astra Zeneca, UK) and Werner Weitschies (University of Greifswald, Germany). The session reviewed where we are today with the development and practical application of clinically relevant specifications and provided a forum for scientists from industry, regulatory agencies, and academia to discuss issues encountered in practice and potential approaches to address these. Paul Dickinson (Seda Pharmaceutical Development Services, UK) described the evolving expectations of the dissolution test, which from humble beginnings has now become the pivotal test linking formulation and process attributes to clinical performance in patients. He described how the drive to put patient benefit at the center of pharmaceutical quality is continuing to gather momentum, with initiatives such as BioRAM (3) seeking to further integrate pharmaceutical quality tests with therapeutic benefit to the patient. He also highlighted some ongoing challenges with implementing clinically relevant specifications, including differing expectations between health authorities globally on how a clinically relevant method and specification should be selected, and the need for increased clarity on the design and interpretation of the clinical studies performed to support these specifications. Sarah Pope Miksinski (FDA, USA) started her talk by describing the patients' expectations of pharmaceutical quality. She emphasized that the potential impact on the patient is at the center of FDA's clinical relevance considerations (safety, efficacy, and supply), which is supported by a broad and robust scientific dialogue during quality review, including consideration of the clinical framework. She also highlighted that one of ONDP's strategic priorities for 2016-2021 is to "enhance robust discussions linking quality to clinical performance," demonstrating FDA's continued commitment to clinical relevance. Following the presentations, the discussion was led by the moderators with Paul Seo (FDA, USA) joining the debate as an additional panel member to give a biopharmaceutics review perspective. A lively discussion followed, covering diverse topics including whether traditional dissolution comparison statistics such as $f_{2}$ testing are still needed if a clinically relevant specification has been established, and what is an appropriate level of discrimination for a dissolution test (i.e., how to appropriately balance clinical relevance and manufacturability considerations). During the debate, Dr. Seo emphasized the importance of making science-based 
decisions and building confidence in them by presenting data and a clear development narrative in the dossier that explains the decisions made and how they relate to the patient. We are not yet at the end of the journey toward establishing clinically relevant specifications as common practice, and expectations and understanding in this area are still evolving as we learn from the day-to-day operation of these methods and specifications and the issues encountered in their development. Open dialogue and collaboration among industry, academia, and regulatory agencies are needed to move this important area forward for the benefit of the patient.

\section{IVRDT FOCUS GROUP}

\section{FACE-TO-FACE MEETING}

The annual focus group face-to-face meeting was held on Monday, October 26. Xujin Lu (BMS, USA) stated the mission and reviewed the accomplishments of the focus group for 2015. Afterward a review of the activities of the subteams on "Technology Improvement and New Dissolution Device Development" (Geoffrey Grove), "Biorelevant/Clinical Relevant Dissolution Method and Specifications" (Jian-Hwa Han), and "CQA Principles for Method Development and Formulation Selection" (Danna Mattocks) was presented. The student outreach activities were presented by Deblina Biswas. The webinars given by Nikoletta Fotaki and Greg Martin to the University of Georgia Student Chapter and University of Florida Student Chapter, respectively, were noted. A Certificate of Appreciation was given to Marie Di Maso (Corealis Pharma, Canada) for her contributions to the Focus Group for the period 2013-2015. Susan D'Souza (Sunovion Pharmaceuticals, USA) became the chair-elect following the elections that took place during the meeting. Finally the 2016 objectives of the focus group and ideas for the programming proposal for the 2016 AAPS Annual Meeting and the sessions of interest for the focus group at the 2015 AAPS Annual Meeting were presented by Nikoletta Fotaki (current chair). A lively discussion by all attendees on proposing ideas and points to consider for future focus group activities followed. The face-to-face meeting of the focus group on November 19, 2015 (BMS, USA) was announced.

\section{REFERENCES}

1. Davit, B.; Braddy, A. C.; Conner, D. P.; Yu, L. X. International Guidelines for Bioequivalence of Systemically Available Orally Administered Generic Drug Products: A Survey of Similarities and Differences. AAPS J. 2013, 15 (4), 974-990. DOI: 10.1208/s12248-013-9499-x.

2. Diaz, D. A.; Colgan, S. T.; Langer, C. S.; Bandi, N. T.; Likar, M. D.; Van Alstine, L. Dissolution Similarity Requirements: How Similar or Dissimilar Are the Global Regulatory Expectations? AAPS J. 2016, 18 (1), 15-22. DOI: 10.1208/s12248-015-9830-9.

3. Selen, A.; Dickinson, P. A.; Müllertz, A.; Crison, J. R.; Mistry, H. B.; Cruañes, M. T.; Martinez, M. N.; Lennernäs, H.; Wigal, T. L.; Swinney, D. C.; Polli, J. E.; Serajuddin, A. T. M.; Cook, J. A.; Dressman, J. B. The Biopharmaceutics Risk Assessment Roadmap for Optimizing Clinical Drug Product Performance. J. Pharm. Sci. 2014, 103 (11), 3377-3397. DOI: 10.1002/ jps.24162. 